\title{
Elevated triglycerides: A matter of the heart and pancreas
}

\author{
D J Blom, MB ChB, MMed, FCP (SA), PhD \\ Division of Lipidology and Hatter Institute for Cardiovascular Research in Africa, Department of Medicine, Faculty of Health Sciences, \\ University of Cape Town, South Africa
}

Corresponding author: D J Blom (dirk.blom@uct.ac.za)

\begin{abstract}
Mild-to-moderate hypertriglyceridaemia (triglyceride (TG) $>1.7-10.0 \mathrm{mmol} / \mathrm{L}$ ) is an independent cardiovascular risk factor, while severe hypertriglyceridaemia (TG $>10.0 \mathrm{mmol} / \mathrm{L}$ ) can cause acute pancreatitis. Hypertriglyceridaemia is polygenic in most patients, and secondary factors, such as uncontrolled diabetes, hypothyroidism, alcohol, renal disease and medication (e.g. corticosteroids, retinoids, oestrogen), often play an important role in precipitating or exacerbating the condition. Patients with mild-to-moderate hypertriglyceridaemia require cardiovascular risk assessment and are in the first instance treated with statins if their cardiovascular risk is high. Control of secondary factors and lifestyle modifications are important in managing residual hypertriglyceridaemia in patients treated with statins. Multiple trials are ongoing to determine whether there is further cardiovascular outcome benefit from adding drugs such as fibrates or omega-3 supplements to the regimen of patients who are receiving statins. Severe hypertriglyceridaemia is managed by removing or controlling secondary factors, following a very low-fat diet and prescribing a fibrate.
\end{abstract}

S Afr Med J 2018;108(4):258-261. DOI:10.7196/SAMJ.2018.v108i4.13235

Of all the lipids routinely measured in clinical practice, serum triglycerides (TGs) are the most variable and can increase more than 50 -fold from baseline. Ideally, fasting TGs should be $<1.7 \mathrm{mmol} / \mathrm{L}$, but TGs can reach values of $>100.0 \mathrm{mmol} / \mathrm{L}$ in some patients. The clinical approach to hypertriglyceridaemia varies considerably, depending on the degree of elevation and the clinical context. Although there are multiple classification schemes for hypertriglyceridaemia, a simple and useful scheme is to classify hypertriglyceridaemia of $>1.7$ $<10.0 \mathrm{mmol} / \mathrm{L}$ as mild to moderate, while TGs $>10.0 \mathrm{mmol} / \mathrm{L}$ are classified as severe hypertriglyceridaemia. ${ }^{[1]}$

\section{What are the potential clinical} consequences of hypertriglyceridaemia? The two most important clinical consequences of hypertriglyceridaemia are acute pancreatitis and atherosclerotic cardiovascular disease (CVD). Severe hypertriglyceridaemia may lead to acute pancreatitis. ${ }^{[2]}$ The mechanisms by which hypertriglyceridaemia causes pancreatitis are not that well understood, but may relate to impairment of capillary perfusion in the pancreas, pancreatic cell necrosis and subsequent release and activation of pancreatic enzymes. When and if pancreatitis will occur in an individual patient is quite unpredictable - some patients may have TGs of $>100.0 \mathrm{mmol} / \mathrm{L}$ and no symptoms, while others develop pancreatitis at much lower TG levels.

Mild-to-moderate hypertriglyceridaemia is an independent cardiovascular risk factor. There is still some debate about the exact mechanisms by which triglyceride-rich lipoproteins (TGRLs) cause atherosclerosis. However, the cholesterol found in these TGRLs is likely a major causative factor. ${ }^{[3]}$ This remnant cholesterol can be approximated by the following calculation:

Remnant cholesterol $=$ total cholesterol - high-density lipoprotein (HDL) cholesterol - low-density lipoprotein (LDL) cholesterol ${ }^{[4]}$

The relative risk of CVD from a $1.0 \mathrm{mmol} / \mathrm{L}$ increase in TGs ranges from 1.14 to 1.80 (dependent on sex and race) after adjustment for other risk factors such as HDL cholesterol. ${ }^{[5]}$
What causes hypertriglyceridaemia?

Hypertriglyceridaemia has multiple and complex causes. Most cases are caused by the interaction of multiple genetic factors (polygenic aetiology) with environmental factors. The relative importance of the genetic and environmental contributions can vary between patients, i.e. in some patients environmental factors may be dominant, while in others genetic factors may be more prominent. Genetic investigation of patients with polygenic hypertriglyceridaemia is difficult because of the multitude of genes that may affect TGs. Variants in these genes individually have very little effect on TGs, but accumulation of many TG-raising variants may lead to hypertriglyceridaemia. ${ }^{[1]} \mathrm{A}$ score that tallies carrier status for TG-raising alleles at 32 genetic loci has been constructed and is higher in patients with hypertriglyceridaemia than in normal controls. ${ }^{[6]}$ Genetic complexity is further increased by gene variants that lower TGs. From a practical point of view, genetic testing of patients with hypertriglyceridaemia is of limited clinical use unless a monogenic cause is strongly suspected.

Monogenic hypertriglyceridaemia is usually severe and manifests in infancy or early childhood. It is usually inherited in an autosomal recessive pattern and is most commonly due to biallelic mutations of the lipoprotein lipase (LPL) gene. LPL deficiency has been described in all South African (SA) population groups, but is most commonly seen in Indians and Afrikaners. Occasionally, patients with dysbetalipoproteinaemia, which results from mutations in apolipoprotein E (apo E), develop severe hypertriglyceridaemia, although the more common phenotype is that of a severe mixed hyperlipidaemia with a total cholesterol to triglyceride ratio of 2:1.

Environmental factors that may contribute to the development of hypertriglyceridaemia include lifestyle factors, medical disorders and drugs (Table 1). Identification and treatment of these secondary factors (where possible) are often key to the management of patients with hypertriglyceridaemia. The clinical experience at Groote Schuur Hospital Lipid Clinic, Cape Town, SA, is that uncontrolled or undiagnosed diabetes is by far the most common secondary factor in patients with hypertriglyceridaemia (D J Blom - unpublished observation, 2018). 


\section{What clinical clues can help to identify patients with hypertriglyceridaemia?}

Mild-to-moderate hypertriglyceridaemia is generally only identified on laboratory testing, although its presence should be suspected in patients with any of the secondary factors (Table 1). Patients with severe hypertriglyceridaemia may develop eruptive or tuboeruptive xanthomata (Fig. 1A and B). Eruptive xanthomata are most commonly found around the elbows, knees, buttocks and thighs and are usually asymptomatic. Lipaemia retinalis (white to creamy appearance of retinal blood vessels) may be visible on fundoscopy. Severe hypertriglyceridaemia cannot be excluded if these physical signs are absent. Laboratory comments referring to a lipaemic specimen' (Fig. 1C) should be taken seriously, as this may be the first clue that a patient has elevated TGs. Lipid screening is often commenced with total cholesterol determination only. If the total cholesterol is very high, it should always be followed up with a full lipogram to detect the patients in whom the very high total cholesterol reflects an accumulation of TGRLs rather than LDL particles.

\begin{tabular}{l} 
Table 1 . Secondary causes of hypertriglyceridaemia \\
\hline Medical disorders \\
Type 2 diabetes/metabolic syndrome \\
Hypothyroidism \\
Renal failure \\
Systemic lupus erythematosus \\
Paraproteinaemia (rare) \\
Lifestyle \\
Obesity \\
Increased alcohol consumption \\
Diet (excess energy, high fat, high glycaemic index) \\
Medication \\
Corticosteroids \\
Retinoids \\
Oestrogen (especially when given orally) \\
Tamoxifen \\
Protease inhibitors (the risk is highest with ritonavir) \\
Cholestyramine \\
Leflunomide \\
Second-generation antipsychotics \\
Cyclosporin \\
Sirolimus \\
Thiazides \\
Beta-blockers \\
Other \\
Pregnancy (in susceptible individuals) \\
Auto-immune states
\end{tabular}

\section{What is the best way to evaluate and investigate patients with hypertriglyceridaemia?}

Once hypertriglyceridaemia has been recognised, the evaluation focuses on identifying secondary factors and assessing the risk of acute pancreatitis or CVD. The majority of hypertriglyceridaemic patients seen in routine clinical practice have identifiable secondary factors that might have precipitated new or exacerbated pre-existing hypertriglyceridaemia. A careful dietary history should be taken, focusing particularly on alcohol intake, fat consumption and refined carbohydrate intake. Collateral history may be particularly helpful when assessing alcohol intake. All medication should be reviewed, including over-the-counter drugs. It is particularly important to enquire specifically about oral contraceptives and other hormonal preparations, as many patients do not regard these as 'medicine'. A family history is also useful and may help to assess the risk of CVD and diabetes. Useful baseline investigations include a fasting glucose, $\mathrm{HbAlC}$ (if appropriate), creatinine, urine dipstick and thyroid-stimulating hormone (TSH). Depending on the clinical situation, liver function testing may be useful to screen for non-alcoholic fatty liver disease. Specialised lipid testing may include agarose gel electrophoresis, gradient gel electrophoresis and determination of apo B and apo A1. The latter investigations should generally be performed at the specialist level, as they are not always straightforward to interpret. Genetic testing plays a minor role in the management of most hypertriglyceridaemic patients and should only be done at a specialist lipid clinic.

There are no algorithms to formally quantify the risk of acute pancreatitis, which is increased in patients with previous pancreatitis and in alcohol users. The risk also increases with the severity of hypertriglyceridaemia, although the correlation is imperfect. Practically, defining all patients with TGs $>10.0 \mathrm{mmol} / \mathrm{L}$ as 'at risk' for acute pancreatitis, is a good policy to ensure rapid assessment and treatment. Cardiovascular risk is assessed using standard clinical guidelines; patients with diabetes and established CVD are classified as very high risk without needing risk calculation. For other patients, risk is quantified using an algorithm such as the Framingham charts. These charts, unfortunately, do not incorporate TGs and may thus underestimate risk in some patients with hypertriglyceridaemia.

\section{How should patients with hypertriglyceridaemia be managed?}

For patients with severe hypertriglyceridaemia, the initial focus is on the prevention of acute pancreatitis, and they should be seen urgently. Any identified secondary factors should be addressed and removed whenever possible. This may include discontinuing and replacing potentially precipitating medications such as corticosteroids,
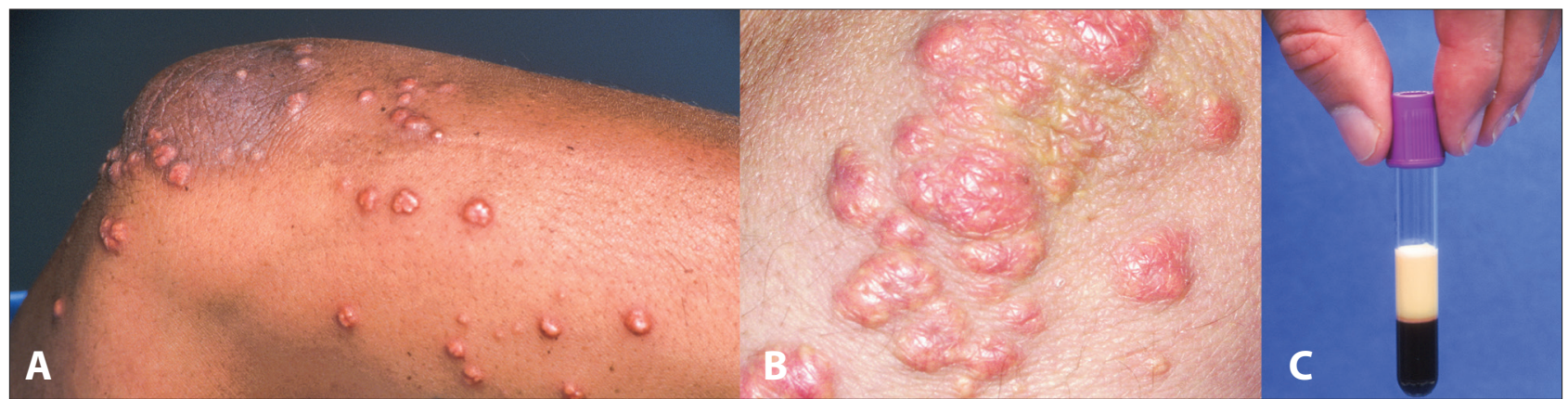

Fig. 1. Clinical signs in hypertriglyceridaemia. (A) Eruptive xanthomata. (B) Tubo-eruptive xanthomata. (C) Lipaemic plasma. 
retinoids or oestrogen-containing preparations when possible to do so. Patients should be advised not to consume alcohol. Improving glycaemic control is an important intervention and, in many patients, it is best and most rapidly achieved either by starting insulin or by intensifying insulin therapy. Dietary restriction of TG intake is very important in most patients with severe hypertriglyceridaemia because poor clearance of TGRL is usually an important component of the pathophysiology. Reducing dietary TG intake reduces the formation of chylomicrons in the intestine and also results in less TG delivery to the liver for incorporation into very low-density lipoproteins (VLDL). Patients with severe hypertriglyceridaemia are often prescribed a diet that is extremely low in dietary fat ('rescue diet': $<10.0 \mathrm{~g}$ of fat per day). Such a diet is not sustainable in the long term, but can lower TGs relatively acutely over a few days. In the long term most patients are maintained on a very low-fat diet $(20-30 \mathrm{~g}$ of dietary fat per day/ 20\% of caloric intake from fat), but the diet can be modified later according to the patient's response and underlying conditions. When referring patients for dietary advice, it is important to specifically request that they not be given a Therapeutic Lifestyle Diet, but that the focus needs to be on reducing dietary fat from all sources. In patients with hypertriglyceridaemia there are no 'good' or 'bad' dietary fats, as all dietary fats (except for special preparations containing medium-chain TGs) are incorporated into chylomicrons and thus contribute to raising serum TGs. For severe hypertriglyceridaemia, fibrates are the most effective drugs, except for patients with monogenic hypertriglyceridaemia, in whom they are usually not effective. In SA, bezafibrate and fenofibrate are the best-known fibrates. These drugs are generally well tolerated, but because they are renally excreted the dose needs to be adjusted in patients with chronic kidney disease. Fibrates can be combined with statins, but this should be done with caution and specialist review is usually worthwhile when considering a statin and fibrate combination. Gemfibrozil should not be combined with a statin because of the risk of drug-drug interactions. Statins are not effective in patients with severe hypertriglyceridaemia, even if prescribed at maximal doses. Using statins as the primary agent in patients with severe hypertriglyceridaemia is a relatively common mistake, because the very high total cholesterol in cases of severe hypertriglyceridaemia (e.g. a total cholesterol of $30.0 \mathrm{mmol} / \mathrm{L}$ in a patient with a TG of $70.0 \mathrm{mmol} / \mathrm{L}$ ) often becomes a misleading focal point. As the concentration of TGRL falls with treatment, the total cholesterol will decrease.

In patients with mild-to-moderate hypertriglyceridaemia, the initial focus is on cardiovascular risk assessment and risk reduction. Very high-risk and high-risk patients require medication in addition to lifestyle modification. Statins are generally the drugs of first choice to reduce cardiovascular risk in patients with mild-tomoderate hypertriglyceridaemia. The statin dose should be adjusted to achieve the guideline-recommended LDL cholesterol target. ${ }^{[7]}$ Non-HDL cholesterol (or apo B) is an important secondary target in patients with hypertriglyceridaemia because it reflects the presence of remnant cholesterol - consider intensifying therapy in patients with LDL cholesterol at target but non-HDL cholesterol above target (the target for non-HDL cholesterol is always $0.8 \mathrm{mmol} / \mathrm{L}$ above the relevant LDL cholesterol target). Important lifestyle interventions include reducing body weight, reducing alcohol intake, reducing simple sugar intake, increasing activity, replacing trans and saturated fats with monounsaturated fats and increasing dietary omega-3 fatty acids.

\section{Should additional therapy be added to a statin to reduce cardiovascular risk further in patients with hypertriglyceridaemia?}

This is currently the source of much debate, and much confusion ensues from different guidelines giving differing advice. There is clinical equipoise, as demonstrated by the approval of several ongoing double-blind placebo-controlled cardiovascular outcomes studies that are evaluating the addition of omega- 3 fatty acids (two studies) or a novel fibrate (one study) to statin-treated patients with residual hypertriglyceridaemia and low HDL cholesterol. Retrospective analyses of fibrate monotherapy studies and a subanalysis of the Action to Control Cardiovascular Risk in Diabetes (ACCORD) study ${ }^{[8]}$ (in which fenofibrate or placebo was added to the regimen of diabetic patients treated with simvastatin) suggest that fibrates may improve cardiovascular outcomes in patients with TGs $>2.3 \mathrm{mmol} / \mathrm{L}$ and low HDL cholesterol. Based on the results of the ACCORD study, the European Society of Cardiology/European Atherosclerosis Society (ESC/EAS) guidelines (on which the South African lipid guidelines are based) (https://www.eas-society.org/) state that addition of fenofibrate to statin-based therapy can be considered in high-risk patients with TGs $>2.3 \mathrm{mmol} / \mathrm{L}$ (class IIB, level C). ${ }^{[7]}$ Once the results of the statin and 'second agent' outcome studies are available, the management of hypertriglyceridaemia in high cardiovascular risk patients will hopefully become much clearer and more evidence based. For now, the emphasis remains on prescribing an adequate statin dose, control of other cardiovascular risk factors and intensive lifestyle modifications. Further information on this topic is available at the website of the Residual Risk Reduction Initiative (http://www.r3i.org/) or the National Lipid Association (https://www.lipid.org).

\section{What novel therapies are in the pipeline?}

The two most promising and advanced novel strategies focus on the inhibition of proteins that interfere with the action of LPL. Lipoprotein lipase (LPL) is directly inhibited by apo C3. Volanesorsen is an antisense oligonucleotide that reduces apo C3 protein production. Encouraging early results have been seen in patients with LPL deficiency and other forms of hypertriglyceridaemia. ${ }^{[9]}$ Angiopoietinlike 3 (ANGPTL-3) is secreted by the liver and also inhibits LPL, particularly after a meal. Monoclonal antibodies against ANGPTL3 are currently in early-phase clinical trials. ${ }^{[10]}$

\section{Conclusion}

Our understanding of the metabolism and clinical role of TGRLs has improved significantly over the past few years. This research has led to the identification of several new and exciting potential therapeutic targets. However, the major challenge in the next few years remains to define how we should optimally use currently available therapies such as fibrates or omega- 3 fatty acids in patients with mild-to-moderate hypertriglyceridaemia and high cardiovascular risk.

\section{Summary}

- Severe hypertriglyceridaemia $(>10.0 \mathrm{mmol} / \mathrm{L})$ can trigger acute pancreatitis.

- A precipitating factor (most commonly uncontrolled or undiagnosed type 2 diabetes mellitus or medication) is present in most patients with severe hypertriglyceridaemia.

- Mild-to-moderate hypertriglyceridaemia $(1.7-10.0 \mathrm{mmol} / \mathrm{L})$ is an independent cardiovascular risk factor. 
- Currently, it is not clear whether a second agent should be added in high cardiovascular risk patients with mild-to-moderate hypertriglyceridaemia, who are being treated with optimised statin therapy.

- Fibrates are the drugs of choice in patients with severe hypertriglyceridaemia.

\section{Acknowledgements. None. \\ Author contributions. Sole author. \\ Funding. None.}

Conflicts of interest. DJB is the national principal investigator for several trials exploring novel treatments for hypertriglyceridaemia. His institution has received payments for conducting clinical trials, trial supervision and his attendance at advisory board meetings from Akcea Therapeutics, AstraZeneca and Kowa Company Ltd.

\footnotetext{
1. Hegele RA, Ginsberg HN, Chapman MJ, et al. The polygenic nature of hypertriglyceridaemia Implications for definition, diagnosis, and management. Lancet Diabet Endocrinol 2014;2(8):655-666. Implications for definition, diagnosis, and man-8
}

2. Whitcomb DC. Clinical practice. Acute pancreatitis. N Engl J Med 2006;354(20):2142-2150. https:// doi.org/10.1056/NEJMcp054958

3. Varbo A, Benn M, Tybjaerg-Hansen A, et al. Remnant cholesterol as a causal risk factor for ischemic heart disease. J Am Coll Cardiol 2013;61(4):427-436. https://doi.org/10.1016/j.jacc.2012.08.1026

4. Nordestgaard BG. Triglyceride-rich lipoproteins and atherosclerotic cardiovascular disease: New insights from epidemiology, genetics, and biology. Circ Res 2016;118(4):547-563. https://doi. org/10.1161/CIRCRESAHA. 115.306249

5. Chapman MJ, Ginsberg HN, Amarenco P, et al. Triglyceride-rich lipoproteins and high-density lipoprotein cholesterol in patients at high risk of cardiovascular disease: Evidence and guidance for management. Eur Heart J 2011;32(11):1345-1361. https://doi.org/10.1093/eurheartj/ehr112

6. Johansen $\mathrm{CT}$, Wang J, McIntyre $\mathrm{AD}$, et al. Excess of rare variants in non-genome-wide association study candidate genes in patients with hypertriglyceridemia. Circ Cardiovasc Genet 2012;5(1):66-72. study candidate genes in patients with hypertriglyceride

7. Catapano AL, Graham I, de Backer G, et al. 2016 ESC/EAS guidelines for the management of dyslipidaemias. Eur Heart J 2016;37(39):2999-3058. https://doi.org/10.1093/eurheartj/ehw272

8. ACCORD Study Group, Ginsberg HN, Elam MB, et al. Effects of combination lipid therapy in type 2 diabetes mellitus. N Engl J Med 2010;362(17):1563-1574. https://doi.org/10.1056/NEJMoa1001282 9. Gaudet D, Alexander VJ, Baker BF, et al. Antisense inhibition of apolipoprotein C-III in patients with hypertriglyceridemia. N Engl J Med 2015;373(5):438-447. https://doi.org/10.1056/NEJMoal400283

0. Stitziel NO, Khera AV, Wang X, et al. ANGPTL3 deficiency and protection against coronary artery disease. J Am Coll Cardiol 2017;69(16):2054-2063. https://doi.org/10.1016/j.jacc.2017.02.030 